\title{
Cyclodextrin-Modified Film Dosage Forms for Oral Candidiasis Treatment
}

\author{
Yoshifumi Murata1 $^{*}$, Kyoko Kofuji', Shushin Nakano², Ryosei Kamaguchi ${ }^{2}$ \\ ${ }^{1}$ Faculty of Pharmaceutical Science, Hokuriku University, Kanazawa, Japan \\ ${ }^{2}$ Morishita Jintan Co. Osaka Technocenter, Hirakata, Japan \\ Email: ${ }^{*}$-murata@hokuriku-u.ac.jp
}

Received 30 March 2015; accepted 10 May 2015; published 13 May 2015

Copyright (C) 2015 by authors and Scientific Research Publishing Inc.

This work is licensed under the Creative Commons Attribution International License (CC BY). http://creativecommons.org/licenses/by/4.0/

(c) (i) Open Access

\begin{abstract}
Oral candidiasis is a common disease in patients with dry mouth. In this study, film dosage forms (FD) incorporating miconazole nitrate, an antifungal agent, were prepared with water-soluble polysaccharide and cyclodextrin (CD). The dissolution profiles of the drug from the FDs were investigated in limited dissolution medium. Soft films were obtained from sodium alginate containing $0.5 \% \alpha$-CD, $\beta$-CD, or $\gamma$-CD. Most FDs were easy to handle, though the film tearing resistance was lower than that of CD-free FDs. Addition of CD to the FD accelerated the drug dissolution rate. Interestingly, this phenomenon was also observed in FDs prepared with pullulan. In contrast, acceleration of the drug dissolution rate was not observed when $C D$ polymer was added to the base solution. The initial drug dissolution rate was controllable by the amount of CD added to the FD. Therefore, FDs prepared with these materials are useful to treat oral candidiasis in patients with dry mouth syndrome.
\end{abstract}

\section{Keywords}

Film Dosage Form, Natural Polysaccharide, Cyclodextrins, Miconazole, Oral Candidiasis

\section{Introduction}

Dry mouth syndrome is a risk factor for oral diseases, including microbial infections, such as dental caries, periodontitis, and candidiasis [1]. Decreased saliva secretion occurs as a symptom of disease, such as diabetes, or as an adverse effect of drug administration [2]. Aging also decreases salivation [3] and can cause difficulty swallowing in the elderly. Saliva plays an important role in cleaning the oral cavity, and has an antimicrobial activity. Oral candidiasis is a fungal infection caused by Candida strains, including Candida albicans. Candida

${ }^{*}$ Corresponding author.

How to cite this paper: Murata, Y., Kofuji, K., Nakano, S. and Kamaguchi, R. (2015) Cyclodextrin-Modified Film Dosage Forms for Oral Candidiasis Treatment. Pharmacology \& Pharmacy, 6, 247-253. http://dx.doi.org/10.4236/pp.2015.65027 
strains are not harmful to healthy individuals, but can cause infections in immunocompromised patients. Oral candidiasis is common in patients with dry mouth [4]. Miconazole nitrate (MCZ) is an antifungal agent widely used to treat Candida infections. It is well known that systemic MCZ administration can cause drug interactionsowing to cytochrome P450 inhibition [5]. Therefore, MCZ is directly applied to the tongue to avoid side effects that occur following gastrointestinal absorption [6]. MCZ gel preparations are commonly used to topically treat oral candidiasis. The patient has to apply the gel evenly in the oral cavity with their tongue. However, this procedure is tedious for patients, and is difficult for elderly patients.

Film is an excellent dosage form for oral care. When film dosage forms (FD) are placed in a small amount of liquid, they swell quickly and release the incorporated drug. FDs prepared with natural polysaccharides, including sodium alginate (ALG) and pullulan (PUL), which are safe for ingestion were previously characterized [7]. These polysaccharides can form films using simple methods that do not require dissolution in organic solvents. For FDs incorporating MCZ, the drug dissolution rate is enhanced by the addition of a surfactant to the base solution [8]. The surfactant used to prepare FDs is selected considering safety, formability, and patient comfort.

Cyclodextrins (CDs) are cyclic oligosaccharides with cone-shaped cavities, which affect the physicochemical properties of organic compounds by forming inclusion complexes. CDs have been studied to enhance the solubility of poorly water-soluble drugs, to improve the stability of unstable compounds, and as a masking agent for medicines and foods [9]-[11]. Some natural CDs are safe for oral administration, particularly $\alpha$-CD, $\beta$-CD, and $\gamma$-CD, which are widely used as food additives [12]. In the present study, FDs were modified by the addition of CD to control the MCZ dissolution rate in limited dissolution medium. Additionally, FDs modified using the adsorbent, cyclodextrin polymer (CDP), were also investigated [13] [14].

\section{Experimental}

\subsection{Materials}

ALG was obtained from NacalaiTesque Inc. (300 cps, Kyoto, Japan), and 1.5\% (w/w) ALG was prepared in deionized water as thefilm base solution. Guluronicacid-rich ALG (IL-6G) was supplied by Kimica Co. (Tokyo, Japan). PUL was supplied by Hayashibara Biochemical Laboratories (Okayama, Japan), and polysaccharides produced by Bifidobacteriumlongum JBL05 (BPS) were supplied by Morishita Jintan (Osaka, Japan). MCZ $\left(\mathrm{C}_{18} \mathrm{H}_{14} \mathrm{C}_{14} \mathrm{~N}_{2} \mathrm{O} \mathrm{HNO}_{3}\right.$, M.W. 479.14), the three CDs $(\alpha-\mathrm{CD}, \beta$-CD, $\gamma$-CD), and the three CDPs $(\alpha-\mathrm{CDP}, \beta$-CDP, $\gamma$-CDP) were obtained fromWako Pure Chemicals (Osaka, Japan). All other chemicals were of reagent grade.

\subsection{FD Preparation}

A CD or CDP was added with agitationto $10 \mathrm{~g}$ of the film base solution.The mixture was thoroughly mixed with sonication, and $3.0 \mathrm{~g}$ of each solution was poured into individual plastic Petri dishes (diameter: $54 \mathrm{~mm}$ ). After $24 \mathrm{~h}$ at $37^{\circ} \mathrm{C}$, the circular films formed on each dish were transferred to a desiccator. Film formation was considered to have failed if a circular film was not obtained, the film had cracks, or the film could not be removed from the bottom of the dish. In the present method, $3 \mathrm{mg}$ of MCZ was theoretically incorporated into each FD.

\subsection{Film Thickness and Rheological Properties}

Film thickness was measured at 10 points on each film using a micrometer (CLM1-15QM; Mitutoyo, Kawasaki, Japan) with a set pressure of $0.5 \mathrm{~N}$. Measurements were made on 3 films, and the mean thickness was calculated for each type. The rheological properties of each film were determined using a rheometer (SUN RHEO TEX SD-700\#; Sun Scientific Co., Tokyo, Japan) at room temperature. The film was fixed on a vial (inner diameter: $1.4 \mathrm{~mm}$; outer diameter: $18.8 \mathrm{~mm}$ ) using a rubber band (Kyowa Co., Osaka, Japan), and was probed with a cylindrical adapter (diameter: $5.0 \mathrm{~mm}$ ). Stress and strain were measured at the point at which the adapter broke through the film. The tests were performed in triplicate.

\subsection{Solubility of MCZ}

The solubility of MCZ was measured in physiological saline containing CD or CDP. MCZ was added to the test solution and shaken at $37^{\circ} \mathrm{C}$ for $24 \mathrm{~h}$. The suspension was removed using a pre-heated plastic syringe (Terumo Co., Tokyo, Japan) at $37^{\circ} \mathrm{C}$ and filtered using asyringe driven filter unit (Millex-HV, pore size: $0.45 \mu \mathrm{m}$, Milli- 
pore Co., Danvers, MA, USA). The solution was diluted with physiological saline and injected onto a high performance liquid chromatography (HPLC) column.

\subsection{Determination of MCZ Content}

The HPLC system (Hitachi Co., Tokyo, Japan) consisted of a pump (L-2130), UV-detector (L-2400), autosampler (L-2200), and chromate-integrator (D-2500) connected to a packed column (150 mm $\times 4.6 \mathrm{~mm}$, Cosmosil 5C $\mathrm{C}_{18}$-MS-II, NacalaiTesque, Kyoto, Japan). To determine the concentration of MCZ, HPLC was conducted at ambient temperature using an eluent consisting of $10 \mathrm{mM} \mathrm{KH}_{2} \mathrm{PO}_{4}$ and acetonitrile (1:4) at a flow rate of $0.8 \mathrm{ml} / \mathrm{min}$ [15]. The detector wavelength was set at $230 \mathrm{~nm}$.

\subsection{MCZ Dissolution Test}

The FD was placed in a plastic dish, and $10 \mathrm{ml}$ dissolution medium (physiological saline preheated to $37^{\circ} \mathrm{C}$ ) was added. The dish was shaken at $300 \mathrm{rpm}$ in a shaker incubator (SI-300; As One Co., Osaka, Japan) at $37^{\circ} \mathrm{C}$. After $1,3,5,10,15,20,30,45$, or 60 minutes, $0.3 \mathrm{ml}$ of the medium was removed using a plastic syringe and filtered through a syringe driven filter unit (pore size: $0.45 \mu \mathrm{m}$ ). Aliquots of the filtered solution (80 $\mu \mathrm{l}$ ) were placed into micro-test tubes $(1.5 \mathrm{ml})$, and $720 \mu \mathrm{l}$ of methanol was added to precipitate the polysaccharide dissolved from the FD. Samples were mixed and centrifuged (7700 $\times$ g, 5 min; H-1300; Kokusan Co., Saitama, Japan), and the supernatants were injected onto the HPLC column. All tests were performed in triplicate.

\section{Results and Discussion}

The polysaccharides used in this study can form thin films, termed FDs, using a casting method. FDs were formed by pouring $1.5 \%$ ALG, $1.2 \%$ BPS, or $4 \%$ PUL containing MCZ $(1 \mathrm{mg} / \mathrm{g})$ into a Petri dish and evaporating the solvent. Addition of CDs to the film base solution affected FD formation. As shown in Figure 1, a circular, soft film was obtained from 1.5\% ALG solutions containing $0.5 \% \alpha$-CD, with a thickness of $43 \mu \mathrm{m}$. FDs were also obtained using ALG containing $0.5 \% \beta$-CD and $0.5 \% \gamma$-CD, as shown in Table 1, although the film

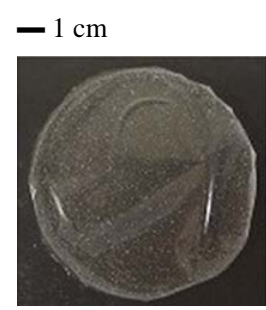

(a)

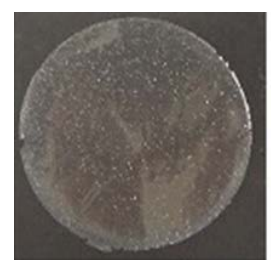

(a) $+0.5 \% \alpha-\mathrm{CD}$

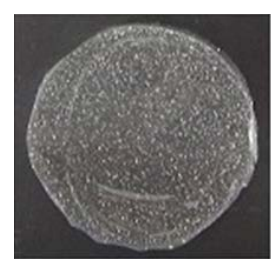

(a) $+0.5 \% \alpha-\mathrm{CDP}$

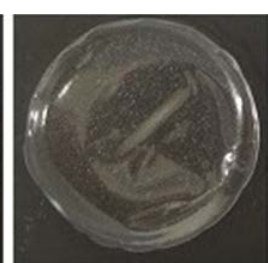

(b)

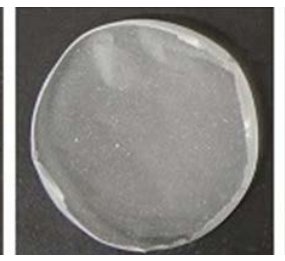

(a) $+0.5 \% \beta$-CD

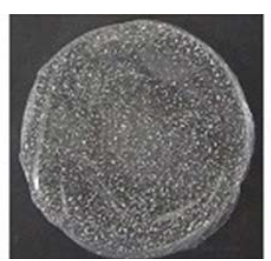

(a) $+0.5 \% \beta$-CDP

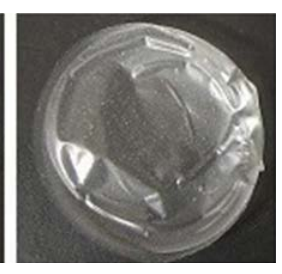

(c)

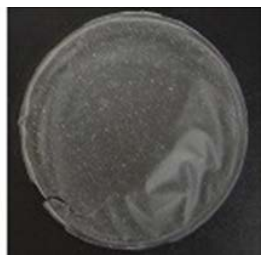

(a) $+0.5 \% \gamma-\mathrm{CD}$

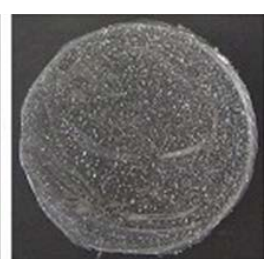

(a) $+0.5 \% \gamma$-CDP

Figure 1. Pictures of FDs prepared with polysaccharides containing MCZ. (a) $1.5 \%$ ALG (b) $4 \%$ PUL (c) $1.2 \%$ BPS. 
prepared with solution containing $0.8 \% \gamma$-CD was cracked. In the case of $1.5 \%$ ALG containing CDP, the FD thickness was approximately $200 \mu \mathrm{m}$. MCZ and other additives were homogeneously dispersed in the FDs, as shown in Figure 2. FDs were also obtained when $4 \%$ PUL containing $0.5 \% \gamma$-CD was used; however, $1.2 \%$ BPS containing $0.5 \%$ CD did not form FDs.

To use FDs incorporating MCZ in oral candidiasis therapy, they must be easy to handle, since the form is directly applied to the target region. Figure 3 shows the additive effect of CD on the rheological properties of FDs prepared with ALG. Although $\gamma$-CD or $\gamma$-CDP lowered the resistance of FDs to tearing, each FD had sufficient strength to be treated by hand.

Since the FD film matrix consists of a water-soluble polysaccharide, it immediately swells in physiological saline at $37^{\circ} \mathrm{C}$, leading to erosion and MCZ release. Figure 4 shows the MCZ dissolution profiles from FDs prepared with $1.5 \%$ ALG. FDs prepared with ALG released $8.8 \% \pm 0.7 \%$ of the incorporated MCZ into the test solution at $5 \mathrm{~min}$. The initial dissolution rate increased following addition of CDs to the film base. For example, $24 \% \pm 8 \%$ of the MCZ was released at 5 min from FDs prepared with ALG containing $0.5 \% \alpha$-CD. Addition of $\beta$-CD or $\gamma$-CD to the FDs also accelerated the drug dissolution rate. This phenomenon was also observed in FDs prepared with the other alginate, IL-6G. However, addition of $0.5 \%$ CDPs to the base solution did not affect the drug dissolution profile. The MCZ dissolution rate increased when CD was added to 4\% PUL. For example, the amount of MCZ dissolved at 5 min increased from 36\% $\pm 4 \%$ to $68 \% \pm 9 \%$ following the addition of $0.5 \%$ $\alpha$-CD to the film base. In addition, the initial MCZ dissolution rate increased when CD-free FD was dipped into the test solution containing $0.15 \% \mathrm{CD}$.

Table 2 shows the additive effect of CD or CDP on MCZ solubility in physiological saline at $37^{\circ} \mathrm{C}$. When $0.15 \% \alpha$-CD was added to the test solution, the solubility increased 1.5 -fold, as compared to $\alpha$-CD free test solution. A similar effect was observed using $\beta$-CD and $\gamma$-CD. In contrast, MCZ solubility was not affected by the addition of $\alpha$-CDP, $\beta$-CDP, or $\gamma$-CDP. CDs can form complexes with some water-insoluble compounds, thereby increasing their solubility. Therefore, the acceleration of MCZ dissolution from FD may be attributable to complex formation between the polysaccharide matrix and CDs in the base solution.

Figure 5 shows the additive effect of $\gamma$-CD on the MCZ dissolution rate from FDs. As the amount of $\gamma$-CD incorporated in the FDs increased, the MCZ dissolution rate increased. To treat oral candidiasis, FDs containing

Table 1. Thickness of FDs prepared with $1.5 \%$ ALG containing $0.5 \%$ additive.

\begin{tabular}{cc}
\hline Additive & Thickness $(\mu \mathrm{m})$ \\
$\alpha-\mathrm{CD}$ & $43 \pm 5$ \\
$\beta$-CD & $45 \pm 6$ \\
$\gamma$-CD & $71 \pm 4$ \\
$\alpha$-CDP & $217 \pm 5$ \\
$\beta$-CDP & $220 \pm 3$ \\
$\gamma$-CDP & $209 \pm 22$ \\
\hline
\end{tabular}

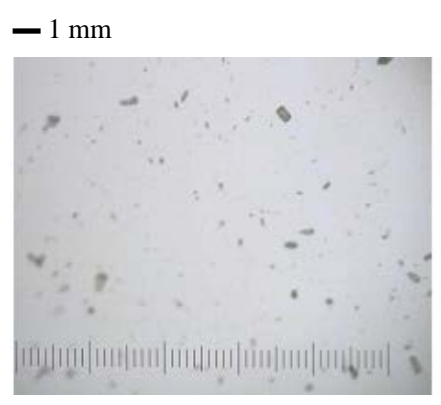

$1.5 \%$ ALG

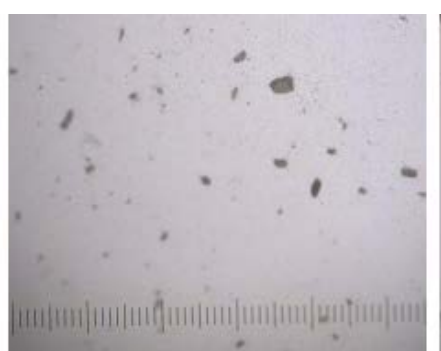

$1.5 \%$ ALG $+0.5 \% \gamma-\mathrm{CD}$

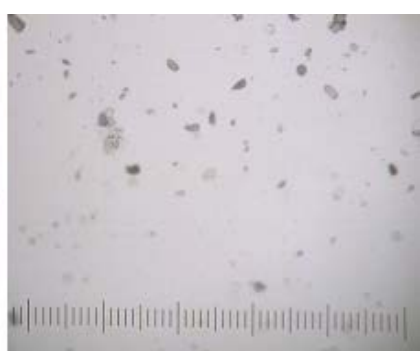

$1.5 \%$ ALG $+0.5 \% \gamma-\mathrm{CDP}$

Figure 2. Stereo-microphotographs of FD surfaces. 


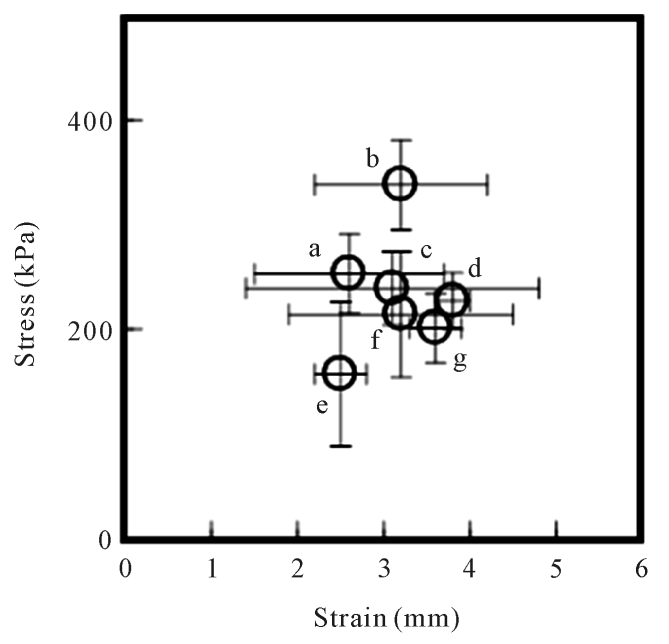

Figure 3. Rheological properties of FDs prepared with 1.5\% ALG containing 0.5\% CD or CDP. (a: additive free, b: $\alpha$-CD, c: $\beta$-CD, d: $\gamma$-CD, e: $\alpha$-CDP, f: $\beta$-CDP, g: $\gamma$-CDP).
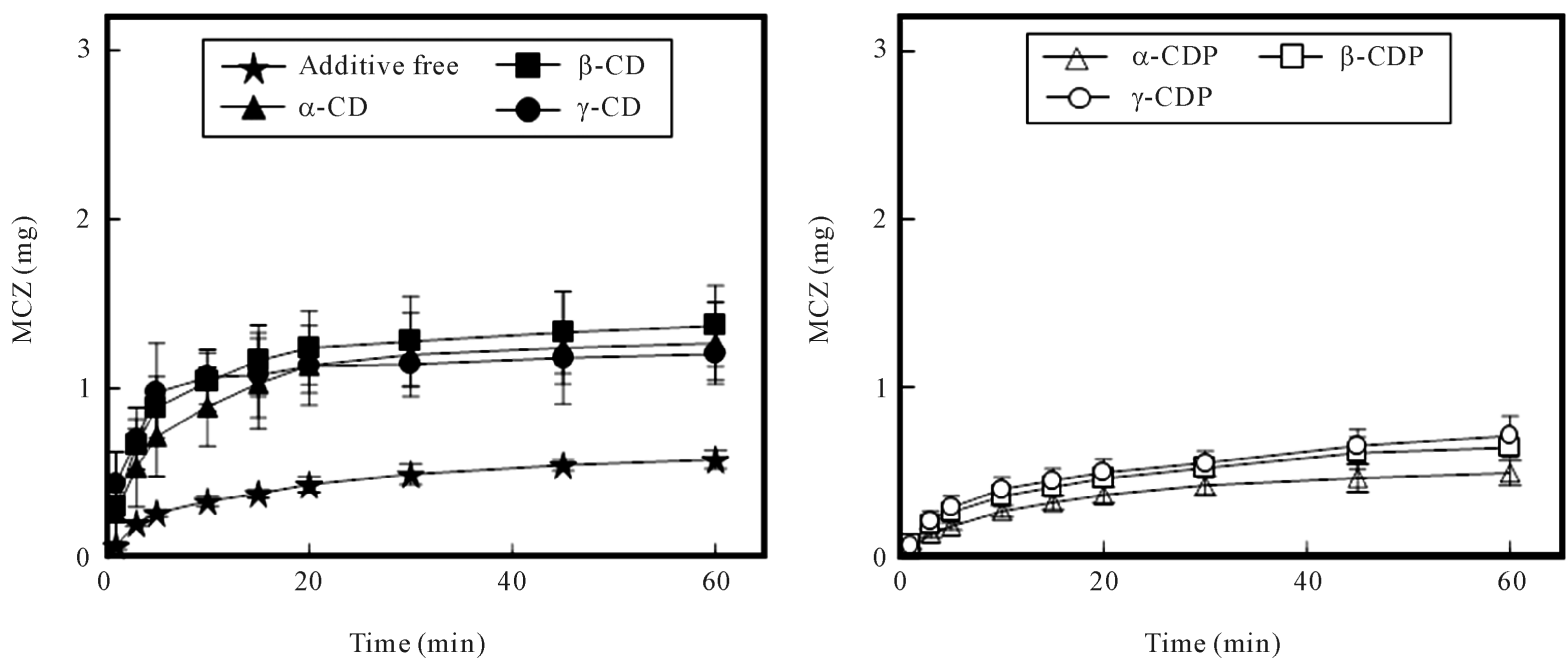

Figure 4. Release profiles of MCZ from FDs prepared with 1.5\% ALG in physiological saline containing CD or CDP.

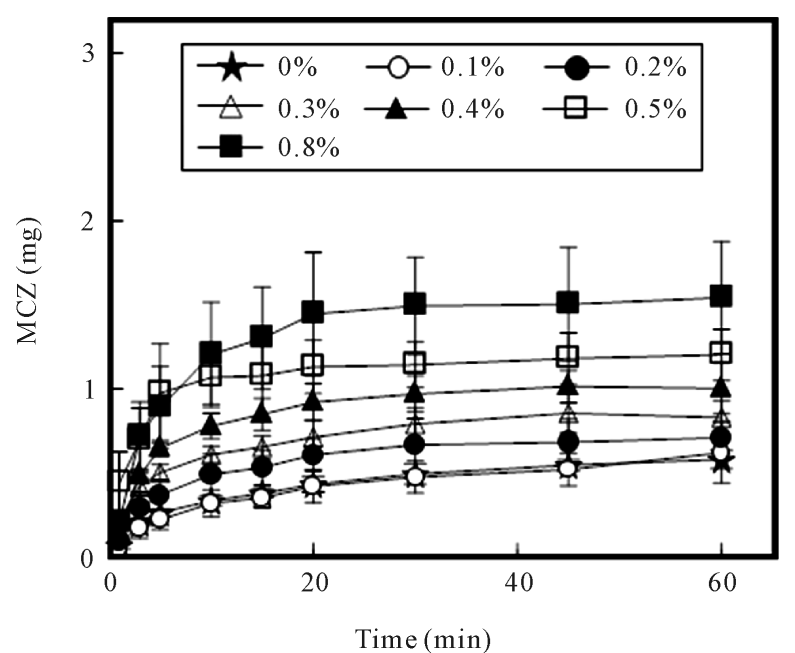

Figure 5. Effect of $\gamma$-CD concentration on MCZ release from FDs. 
Table 2. Solubility of MCZ in physiological saline containing $0.15 \%$ additive at $37^{\circ} \mathrm{C}$.

\begin{tabular}{cc}
\hline Additive & Solubility $(\mathrm{mM})$ \\
\hline additive free & 1.2 \\
$\alpha$-CD & 1.8 \\
$\beta$-CD & 1.8 \\
$\gamma$-CD & 1.9 \\
$\alpha$-CDP & 1.1 \\
$\beta$-CDP & 1.2 \\
$\gamma$-CDP & 1.1 \\
\hline
\end{tabular}

*Suspension.

MCZ are administered in the oral cavity to act directly at the affected site. These data show that the amount of MCZ that reached the site per unit time could be controlled by the addition of CDs.

In this study, FDs were prepared with polysaccharides and CDs incorporating MCZ. All materials used are safe for oral administration. When treating oral candidiasis, the antifungal agent must be applied repeatedly to completely treat the disease [16]. Therefore, the dissolution rate of MCZ in saliva affects the antifungal activity. FD easily disintegrates upon contact with a small amount of saliva, allowing it to deliver the incorporated MCZ to the affected part of the oral cavity. The MCZ dissolution rate can be controlled by the amount of CD added to the base FD solution. Thus, FDs prepared with water-soluble polymers, such as polysaccharides, are useful for treating localized problems in the oral cavity and simplify the administration of drugs to patients. This preparation is a candidate for the treatment of oral candidiasis caused by dry mouth syndrome.

\section{Acknowledgements}

This work was supported in parts by a grant from The OTC Self-Medication Promotion Foundation (2014).

\section{References}

[1] Han, P., Suarez-Durall, P. and Mulligan, R. (2015) Dry Mouth: A Critical Topic for Older Adult Patients. Journal of Prosthodontic Research, 59, 6-19. http://dx.doi.org/10.1016/j.jpor.2014.11.001

[2] NoboruKuroiwa, D., Ruiz Da Cunha Melo, M.A., Balducci, I., Bortolin Lodi, K., Ghislaine Oliveira Alves, M. and Dias Almeida, J. (2014) Evaluation of Salivary Flow and Drug Interactions in Patients with a Diagnosis of Diabetes Mellitus. Minerva Stomatologica, 63, 421-426.

[3] Gonsalves, W.C., Wrightson, A.S. and Henry, R.G. (2008) Common Oral Conditions in Older Persons. American Family Physician, 78, 845-852.

[4] Garcia-Cuesta, C., Sarrion-Pérez, M.G. and Bagán J.V. (2014) Current Treatment of Oral Candidiasis: A Literature Review. Journal of Clinical and Experimental Dentistry, 6, 576-582. http://dx.doi.org/10.4317/jced.51798

[5] Niwa, T., Imagawa, Y. and Yamazaki, H. (2014) Drug Interactions between Nine Antifungal Agents and Drugs Metabolized by Human Cytochromes P450. Current Drug Metabolism, 15, 651-679. http://dx.doi.org/10.2174/1389200215666141125121511

[6] Gronlund, J., Saari, T.I., Hagelberg, N., Neuvonen, P.J., Olkkola, K.T. and Laine, K. (2011) MiconazoleOral Gel Increases Exposure to Oral Oxycodone by Inhibition of CYP2D6 and CYP3A4. Antimicrobial Agents and Chemotherapy, 55, 1063-1067. http://dx.doi.org/10.1128/AAC.01242-10

[7] Murata, Y., Kofuji, K., Nishida, N. and Kamaguchi, R. (2012) Development of Film Dosage Form Containing Allopurinol for Prevention of Oral Mucositis.International Scholarly Research Network Pharmaceutics, 2012, Article ID: 764510. http://dx.doi.org/10.5402/2012/764510

[8] Murata, Y., Isobe, T., Kofuji, K., Nishida, N. and Kamaguchi, R. (2013) Development of Film Dosage Forms Containing Miconazole for the Treatment of Oral Candidiasis. Pharmacology \& Pharmacy, 4, 325-330. http://dx.doi.org/10.4236/pp.2013.43047

[9] Stella, V.J. and He, Q. (2008) Cyclodextrins. Toxicologic Pathology, 36, 30-42. 
http://dx.doi.org/10.1177/0192623307310945

[10] Tamamoto, L.C., Schmidt, S.J. and Lee, S.Y. (2010) Sensory Properties of Ginseng Solutions Modified by Masking Agents. Journal of Food Science, 75, 341-347. http://dx.doi.org/10.1111/j.1750-3841.2010.01749.x

[11] Ogawa, N., Takahashi, C. and Yamamoto, H. (2015) Physicochemical Characterization of Cyclodextrin-Drug Interactions in the Solid State and the Effect of Water on these Interactions. Journal of Pharmaceutical Science, 104, 942-954. http://dx.doi.org/10.1002/jps.24319

[12] Irie, T. and Uekama, K. (1997) Pharmaceutical Applications of Cyclodextrins. III. Toxicological Issues and Safety Evaluation. Journal of Pharmaceutical Science, 86, 147-954. http://dx.doi.org/10.1021/js960213f

[13] García-Fernández, M.J., Tabary, N., Martel, B., Cazaux, F., Oliva, A., Taboada, P., Concheiro, A. and Alvarez-Lorenzo, C. (2013) Poly-Cyclodextrins as Ethoxzolamide Carriers in Ophthalmic Solutions and in Contact Lenses. Carbohydrate Polymers, 98, 1343-1352. http://dx.doi.org/10.1016/j.carbpol.2013.08.003

[14] Simões, S.M., Veiga, F., Ribeiro, A.C., Figueiras, A.R., Taboada, P., Concheiro, A. and Alvarez-Lorenzo, C. (2014) Supramolecular Gels of Poly- $\alpha$-Cyclodextrin and PEO-Based Copolymers for Controlled Drug Release. European Journal of Pharmaceutics and Biopharmaceutics, 87, 579-588. http://dx.doi.org/10.1016/j.ejpb.2014.04.006

[15] Pershing, L.K., Corlett, J. and Jorgensen, C. (1994) In Vivo Pharmacokinetics and Pharmacodynamics of Topical Ketoconazole and Miconazole in Human Stratum Corneum. Antimicrobial Agents and Chemotherapy, 38, 90-95. http://dx.doi.org/10.1128/AAC.38.1.90

[16] Garcia-Cuesta, C., Sarrion-Pérez, M.G. and Bagán, J.V. (2014) Current Treatment of Oral Candidiasis: A Literature Review. Journal of Clinical and Experimental dentistry, 6, 576-582. http://dx.doi.org/10.4317/jced.51798 